\title{
RATIONAL CURVES ON HOLOMORPHIC SYMPLECTIC FOURFOLDS
}

\author{
by
}

Brendan Hassett and Yuri Tschinkel

\section{Introduction}

One of the main problems in the theory of irreducible holomorphic symplectic manifolds is the description of the ample cone in the Picard group. The goal of this paper is to formulate explicit Hodge-theoretic criteria for the ampleness of line bundles on certain irreducible holomorphic symplectic manifolds. It is well known that for K3 surfaces the ample cone is governed by $(-2)$-curves. More generally, we expect that certain distinguished twodimensional homology classes of the symplectic manifold should correspond to explicit families of rational curves, and that these govern its ample cone.

The program for analyzing the ample cone of a symplectic manifold divides naturally into three parts. First, for each deformation type of irreducible holomorphic sympectic manifolds we identify distinguished Hodge classes in $H_{2}(\mathbb{Z})$ that should be represented by rational curves. We consider $H_{2}(\mathbb{Z})$ and $H^{2}(\mathbb{Z})$ as quadratic lattices with respect to a natural quadratic form (the Beauville form discussed in Section 2) and distinguish orbits in $H_{2}(\mathbb{Z})$ under the orthogonal group. These orbits are often characterized by the 'squares' of the corresponding elements, i.e., the value of the quadratic form. These distinguished classes should be in one-to-one correspondence with certain geometrically described rational curves on $F$. In many cases, one can use deformation arguments to show that, if a given distinguished class represents a rational curve of a certain type then this remain true under deformation (see Section 4). Second, one shows that rational curves with the geometry 
described govern the ample cone of $F$. This entails classifying possible contractions of symplectic manifolds - a very active topic of current research (see the discussion of the literature below) - and interpretting this classification in terms of the numerical properties of the contracted curves. It also involves the classification of base loci for sections of line bundles on a symplectic manifold. The third part of the program is to show that a divisor class satisfying certain numerical conditions arises from a big line bundle and thus yields a birational transformation of the symplectic manifold. This aspect of the program is still largely conjectural; see the work of Huybrechts cited below.

We are mainly concerned with the first step of this program in a specific case. Let $F$ be an irreducible holomorphic symplectic fourfold deformation equivalent to the punctual Hilbert scheme $S^{[2]}$ for some K3 surface $S$. Given the Hodge structure on $H^{2}(F)$, we describe explicitly (but conjecturally) the cone of effective curves on $F$ and, by duality, the ample cone of $F$. As in the case of $\mathrm{K} 3$ surfaces, each divisor class of square -2 induces a reflection preserving the Hodge structure. The 'birational ample cone' is conjectured to be the interior of a fundamental domain for this reflection group. However, the ample cone may be strictly smaller than the birational ample cone, owing to the existence of elementary transformations along $\mathbb{P}^{2}$ 's in $F$. The corresponding classes have square -10 with respect to the Beauville form.

Here we give a brief and incomplete overview of work on related problems. Wilson has studied Calabi-Yau threefolds from a similar point of view (see [28],[29],[30], and [31].) If $F$ is a Calabi-Yau threefold then the Picard group of $F$ is equipped with two integer-valued forms: a cubic form $\mu$ (the intersection form) and a linear form $c_{2}(F)$ (obtained by intersecting with the second Chern class of the tangent bundle.) Wilson gives criteria for the existence of birational contractions and elliptic fibrations in terms of the number-theoretic properties of these forms.

Namikawa [22] and Shepherd-Barron [25], have proven structural results on the geometry of birational morphisms from holomorphic symplectic manifolds. There are also results in this direction by Burns, Hu, and Luo [7] and Wierzba [27]. Matsushita [19] [20] has proven a detailed description of fiber structures on irreducible holomorphic symplectic manifolds. Huybrechts has conjectured a projectivity criterion for irreducible holomorphic symplectic manifolds and elaborated consequences of his criterion. See [13] and the erratum in [14]; we will be careful to distinguish results fully proved in [13] 
from those which remain conjectural. Markman [17] has developed a theory of generalized elementary transformations for moduli spaces of sheaves on K3 surfaces.

Our study of rational curves on symplectic manifolds began with the detailed study of a particular example: the variety $F$ parametrizing lines on a cubic fourfold $X$ is an irreducible holomorphic symplectic manifold. The existence of rational curves on $F$ coincides with the presence of rational ruled surfaces on $X$. Our conjectures therefore shed light on the effectivity of certain codimension-two cycles on $X$. Conversely, the projective geometry of cubic fourfolds provides a useful laboratory where we may test our claims. As an application of our conjectures, we find that the presence of distinguished Hodge classes on $X$ often implies the existence of special unirational parametrizations of $X$.

This paper is organized as follows. In Section 2 we recall basic results and conjectures for irreducible holomorphic symplectic manifolds. In the next section, we introduce the notion of nodal classes and state our basic conjectures. In Section 4 we give some deformation-theoretic evidence for our conjectures. The rest of the paper is devoted to examples supporting the conjectures. Section 5 is devoted to Hilbert schemes $S^{[2]}$ for K3 surfaces of small degree. We describe examples of nonnodal rational curves and certain codimension-two behavior in Section 6. We turn to the projective geometry of cubic fourfolds in the last section. Questions of rationality and unirationality are addressed in Section 7.5.

Throughout, we work over $\mathbb{C}$. A primitive element of an abelian group $A$ is one that cannot be written in the form $n x$ for any $x \in A$ or $n \in \mathbb{Z}, n>1$. An indecomposable element of a monoid is an element which cannot be written in the form $a+b$ for some nonzero $a$ and $b$ in this monoid. Recall that an element $v$ of a convex real cone $C$ is an extremal ray if, for any $u, w \in C$ with $u+w=v$ we necessarily have $u, w \in \mathbb{R}_{+} v$.

Acknowledgements. The first author was partially supported by an NSF postdoctoral fellowship, an NSF continuing research grant, and The Institute of Mathematical Sciences at the Chinese University of Hong Kong. The second author was partially supported by the NSA. We thank D. Matsushita and Y. Namikawa for sending us their preprints. Our treatment of rationality 
questions in Section 7.5 benefitted from discussions with D. Saltman and I. Dolgachev.

\section{Generalities}

Let $F$ be an irreducible, holomorphic symplectic manifold of dimension $2 n$. This means that $F$ is compact, Kähler, simply connected, and $H^{0}\left(F, \Omega_{F}^{2}\right)$ is spanned by an everywhere-nondegenerate 2-form (cf. [2] and [6]). The second cohomology group $H^{2}(F, \mathbb{Z})$ carries a nondegenerate integer-valued quadratic form $($,$) , the Beauville form. It has signature \left(3, b_{2}(F)-3\right)$ and its restriction to $H^{1,1} \cap H^{2}(F, \mathbb{Z})$ has signature $\left(1, b_{2}(F)-3\right)$, where $b_{2}(F)$ is the second Betti number (see [3] and [13] $§ 1.9$ for more details).

Using the universal coefficient theorem, we extend the Beauville form to a $\mathbb{Q}$-valued form on $H_{2}(F, \mathbb{Z})$. Concretely, given some primitive $R \in H_{2}(F, \mathbb{Z})$, there exists a unique class $w \in H^{2}(F, \mathbb{Q})$ such that $R v=(w, v)$ for all $v \in H^{2}(F, \mathbb{Z})$. We set $(R, R)=(w, w)$. Let $\rho \in H^{2}(F, \mathbb{Z})$ denote the primitive class such that $c \rho=w$ for some $c>0$. Note that $R$ is of type $(2 n-1,2 n-1)$ iff $\rho$ is of type $(1,1)$. Conversely, given a primitive $\rho \in$ $H^{2}(F, \mathbb{Z})$ with $\left(\rho, H^{2}(F, \mathbb{Z})\right)=d \mathbb{Z}$ and $d>0$, there exists a primitive class $R \in H_{2}(F, \mathbb{Z})$ with $d R v=(v, \rho)$ for all $v \in H^{2}(F, \mathbb{Z})$.

Throughout this paper, the square of a divisor class means the square with respect to the Beauville form. In the sequel we will assume that $F$ has a polarization $g$; note that $(g, g)>0$ [13] $\S 1.9$. Denote by

$$
\operatorname{Pic}_{+}(F, g)=\{v \in \operatorname{Pic}(F) \mid(v, g)>0\}
$$

the positive halfspace (with respect to $g$ and the Beauville form). Let $\Lambda_{+}(F, g) \subset$ $\operatorname{Pic}_{+}(F, g)$ be the vectors with positive square.

We denote by $\Lambda_{\text {amp }}(F)$ and $\Lambda_{\text {nef }}(F)$ the monoids of ample and nef divisor classes. Let $N_{1}(F)$ be the group of classes of 1-cycles (up to numerical equivalence), $N E(F) \subset N_{1}(F)_{\mathbb{R}}$ the cone of effective curves, and $\overline{N E}(F)$ its closure. We denote by $\Lambda_{+}^{*}(F, g)$ the set of classes $R$ such that the corresponding $\rho$ is contained in $\Lambda_{+}(F, g)$.

We next review properties of line bundles on polarized irreducible holomorphic symplectic manifolds which follow from standard results of the minimal model program. 
Proposition 2.1. - Let $(F, g)$ be a polarized irreducible holomorphic symplectic manifold.

1. A class $\lambda \in \operatorname{Pic}(F)$ is ample iff $\lambda \in \Lambda_{+}(F, g)$ and $\lambda C>0$ for each curve $C \subset F$ (see [13] Cor. 6.4).

2. Any class $\lambda \in \Lambda_{\text {nef }}(F)$ which is big has the property that the line bundle $\mathcal{L}(\lambda)$ has no cohomology and $\mathcal{L}(m \lambda)$ is globally generated for $m \gg 0$; it therefore defines a birational morphism $b: F \rightarrow Y$ (see [15], Remark 3-1-2 and Theorem 1-2-3).

The following statements were conjectured by Huybrechts [14] (but stated as Theorem 3.11 and Corollary 3.10 in [13] with an incomplete proof):

CONJECTURe 2.2. - Let $F$ be an irreducible holomorphic symplectic manifold.

1. $F$ is projective iff there exists a class $g \in \operatorname{Pic}(F)$ with $(g, g)>0$.

2. If $g$ is a polarization for $F$ then any class $\lambda \in \Lambda_{+}(F, g)$ is big.

Now assume $F=S^{[n]}$, the Hilbert scheme of length $n$ subschemes of a K3 surface $S$. Then we have an isomorphism

$$
\operatorname{Pic}\left(S^{[n]}\right) \simeq \operatorname{Pic}(S) \oplus_{\perp} \mathbb{Z} e
$$

compatible with the Beauville form (see [3]). Each divisor $f$ on $S$ determines a divisor on $S^{[n]}$, also denoted by $f$, and corresponding to the subschemes with some support in $f$. The locus of subschemes with support at fewer than $n$ points has class $2 e$ and $(e, e)=-2(n-1)$.

More generally, if $F$ is deformation equivalent to $S^{[n]}$ then the Beauville form on $L:=H^{2}(F, \mathbb{Z})$ is an even, integral form isomorphic to

$$
U^{\oplus 3} \oplus_{\perp}\left(-E_{8}\right)^{\oplus 2} \oplus_{\perp} \mathbb{Z} e,
$$

where $U$ is a hyperbolic plane, $E_{8}$ the positive-definite quadratic form associated to the corresponding Dynkin diagram.

Proposition 2.3. - Assume that $n=2$ so that

$$
L \simeq U^{\oplus 3} \oplus_{\perp}\left(-E_{8}\right)^{\oplus 2} \oplus_{\perp}(-2) .
$$

The orbits of primitive elements $v \in L$ under the action of $\Gamma=\operatorname{Aut}(L)$ are classified by $(v, v)$ and the ideal $(v, L)$ (which equals $\mathbb{Z}$ or $2 \mathbb{Z}$.) 
Proof. We shall classify primitive imbeddings of the lattice $K:=\mathbb{Z} v=$ $(2 d)$ (where $(v, v)=2 d$ ) into $L$. For simplicity, we first restrict to the case $d \neq$ 0 . The basic technical tool is the discriminant group $d(L):=L^{*} / L$ and the associated $\mathbb{Q} / 2 \mathbb{Z}$-valued quadratic form $q_{L}$ [23]. We have $d(L) \simeq \mathbb{Z} / 2 \mathbb{Z}$ with $q_{L}$ equal to $-\frac{1}{2}(\bmod 2 \mathbb{Z})$ on the generator. Let $K^{\perp}$ denote the orthogonal complement to $K$ in $L, d(K)$ and $d\left(K^{\perp}\right)$ the discriminant groups, and $q_{K}$ and $q_{K^{\perp}}$ the corresponding forms, so that $d(K) \simeq \mathbb{Z} / 2 d \mathbb{Z}$ with $q_{K}$ equal to $\frac{1}{2 d}$ $(\bmod 2 \mathbb{Z})$ on the generator $\frac{v}{2 d}$. We have the sequence of inclusions

$$
K \oplus K^{\perp} \subset L \subset L^{*} \subset K^{*} \oplus\left(K^{\perp}\right)^{*}
$$

$L^{*}$ consists of the elements of $K^{*} \oplus\left(K^{\perp}\right)^{*}$ which are integral on $L$. Let $H$ (resp. $H^{*}$ ) be the image of $L$ (resp. $L^{*}$ ) in $d(K) \oplus d\left(K^{*}\right)$, so that $d(L)=$ $H^{*} / H$. Note that $H$ is isotropic and $H^{*}$ is the annihilator of $H$ with respect to $q_{K} \oplus q_{K^{\perp}}$ (or the $\mathbb{Q} / \mathbb{Z}$-valued bilinear form associated to it). The projection of $H$ into $d(K)$ is injective and its image is a subgroup of index one or two, depending on whether $(v, L)=\mathbb{Z}$ or $2 \mathbb{Z}$.

In the first case, $d\left(K^{\perp}\right)$ contains the projection of $H$ as an index two subgroup. Since $H$ is isotropic for $q_{K} \oplus q_{K^{\perp}}$, the restriction $q_{K^{\perp}} \mid H$ takes value $-\frac{1}{2 d}(\bmod 2 \mathbb{Z})$ on the generator. Let $x \in H^{*}$ be a nontrivial element projecting to 0 in $d(K)$, which may be regarded as an element of $d\left(K^{\perp}\right)$. We have $q_{K^{\perp}}(x)=-\frac{1}{2}(\bmod 2 \mathbb{Z})$ because $x$ generates $d(L)$. Since $x$ annihilates $H$, it follows that $d\left(K^{\perp}\right)=H+\mathbb{Z} x \simeq \mathbb{Z} / 2 d \mathbb{Z} \oplus_{\perp} \mathbb{Z} / 2 \mathbb{Z}$. This determines the discriminant form (and signature) of $K^{\perp}$ completely, which determines it up to isomorphism [23] 1.14.2. The classification of the primitive imbeddings $K \hookrightarrow L$ follows from [23] 1.15.1.

The proof in the second case is similar but easier, as $d\left(K^{\perp}\right)$ is equal to $H$, so its discriminant form is easily computed.

If $d=0$, we may produce an element $e^{\prime} \in L$ with $\left(v, e^{\prime}\right)=0,\left(e^{\prime}, e^{\prime}\right)=-2$, and $\left(e^{\prime}, L\right)=2 \mathbb{Z}$. By our previous argument $e^{\prime} \in \Gamma e$, so we may assume that $w \in U^{\oplus 3} \oplus\left(-E_{8}\right)^{\oplus 2}$. Then the result follows from [16] $\S 2$.

When $n=2$, we have an identity $c_{2}(F) \cdot v \cdot v=30(v, v)$ (given in [13] 1.11 up to a multiplicative constant). Hence Riemann-Roch takes the form

$$
\chi(F, \mathcal{L}(v))=\frac{1}{8}((v, v)+4)((v, v)+6) .
$$




\section{Conjectures}

In this section $(F, g)$ is a $g$-polarized irreducible holomorphic symplectic manifold, deformation equivalent to the Hilbert scheme of length-two subschemes of a K3 surface. Let $E$ be the (possibly infinite!) set of classes $\rho \in \operatorname{Pic}_{+}(F, g)$ satisfying one of the following:

1. $(\rho, \rho)=-2$ and $(\rho, L)=2 \mathbb{Z}$,

2. $(\rho, \rho)=-2$ and $(\rho, L)=\mathbb{Z}$,

3. $(\rho, \rho)=-10$ and $(\rho, L)=2 \mathbb{Z}$,

Let $E^{*}$ be the corresponding classes $R \in H_{2}(F, \mathbb{Z})$; this means that for some $\rho \in E$ we have

$$
(v, \rho)= \begin{cases}R v & \text { where }(\rho, L)=\mathbb{Z} \\ 2 R v & \text { where }(\rho, L)=2 \mathbb{Z}\end{cases}
$$

for each $v \in L$. In particular, $R$ satisfies one of the following

1. $(R, R)=-\frac{1}{2}$,

2. $(R, R)=-2$,

3. $(R, R)=-\frac{5}{2}$.

Let $N_{E}(F, g) \subset H_{2}(F, \mathbb{Z})$ be the smallest real cone containing $E^{*}$ and the elements $R \in N_{1}(F)$ such that $R \cdot g>0$ and the corresonding $\rho$ has nonnegative square. Note that the boundary of $N_{E}(F, g)$ is polyhedral in a neighborhood of any boundary point with negative square. This follows from the fact that the Beauville form is negative definite on the orthogonal complement to $g$ in $\operatorname{Pic}(F)$.

We now can state our main conjecture.

CONJECTURE 3.1 (Effective curves conjecture). -

$$
N E(F)=N_{E}(F, g) \text {. }
$$

The analogous theorem for K3 surfaces may be found in $\S 1.6$ and 1.7 of [16] (see also [4]).

The classes in $E^{*}$ that are extremal in (the closure of) $N_{E}(F, g)$ will be called nodal classes, in analogy with the terminology for K3 surfaces (see [16], Section 1.4). The nodal classes are denoted $E_{\text {nod }}^{*}$ and the corresponding classes in $E$ are denoted $E_{\text {nod. }}$. Since the boundary of $N_{E}(F, g)$ is polyhedral in a neighborhood of any nodal class, it follows that a class $R \in E^{*}$ is nodal iff no positive multiple of $R$ is decomposable. 
REMARK 3.2. - Consider the monoid $N_{E}(F, g) \cap H_{2}(F, \mathbb{Z})$. In contrast to the situation for K3 surfaces, it is possible for a $(-2)$-class in the monoid to be indecomposable but not nodal. Indeed, there exist examples with $\operatorname{rk} N_{1}(F)=$ 2 .

Conjecture 3.1 and Proposition 2.1 yield a characterization of the ample monoid:

$$
\begin{aligned}
\Lambda_{\mathrm{amp}}(F)= & \left\{\lambda \in \Lambda_{+}(F, g):(\lambda, v)>0\right. \text { for each } \\
& \left.v \in \operatorname{Pic}_{+}(F, g) \text { with }(v, v) \geq 0 \text { or } v \in E\right\} .
\end{aligned}
$$

The signature of the Beauville form implies that if $(\lambda, \lambda)$ and $(v, v)$ are both nonnegative then $(\lambda, v) \geq 0$; indeed if $\lambda$ and $v$ are linearly independent then strict inequality follows. Furthermore, verifying the positivity condition against nonextremal classes is clearly redundant. We therefore obtain the following simplification:

CONJECTURe 3.3 (Consequence of Conjecture 3.1). -

$$
\Lambda_{\mathrm{amp}}(F)=\left\{\lambda \in \Lambda_{+}(F, g):(\lambda, v)>0 \text { for each } v \in E_{\text {nod }}\right\} .
$$

This generalizes Proposition 1.9 of [16].

We digress to consider the monoid of effective divisors. Its description is the same as for K3 surfaces.

CONJECTURE 3.4 (Effective divisors conjecture). - The monoid of effective divisors is generated by the elements of $\operatorname{Pic}_{+}(F, g)$ with square $\geq-2$.

REMARK 3.5. - A (-2)-class is extremal in the (conjectured) cone of effective divisors iff it is indecomposable in the (conjectured) monoid of effective divisors. We expect that each of these $(-2)$-classes is realized by a conic bundle over a K3 surface.

We next discuss classes $\lambda \in \Lambda_{\text {nef }}(F)$, i.e., those in the boundary of the ample cone. Conjecture 2.2 implies that when $(\lambda, \lambda)>0$ the sections of $\mathcal{L}(m \lambda)$ for $m \gg 0$ give a birational morphism $b: F \rightarrow Y$. Any curve represented by a nodal class $R \in E_{\text {nod }}^{*}$ orthogonal to $\lambda$ is contracted by $b$.

Conjecture 3.6 (Nodal classes conjecture). - Each nodal class $R \in E_{\text {nod }}^{*}$ represents a rational curve contracted by a birational morphism $b$ given by 
sections of $\mathcal{L}(m \lambda)$, where $\lambda$ is any class on the boundary of the ample cone satisfying $\lambda R=0$.

1. If $(R, R)=-\frac{1}{2},-2$ (i.e., the corresponding $\rho$ is a (-2)-class) then $\rho$ is represented by a family of rational curves parametrized by a K3 surface. This family can be blown down to rational double points.

2. If $(R, R)=-\frac{5}{2}$ (i.e., the corresponding $\rho$ is a $(-10)$-class) then $\rho$ is represented by a family of lines contained in a plane $\mathbb{P}^{2}$. This plane can be contracted to a point.

The following theorem of Namikawa provides support for this conjecture (part of the theorem was proved first by Shepherd-Barron [25] and related results were obtained by Wierzba [27]).

Theorem 3.7. - ([22] Props. 1.1 and 1.4, [25]) Let $b: F \rightarrow Y$ be a birational projective morphism from a projective holomorphic symplectic manifold to a normal variety.

1. There exists a subvariety $Z \subset Y$ of codimension at least four, so that $Y \backslash Z$ is singular along a smooth codimension-two subvariety $S$, which admits a nondegenerate holomorphic two-form. Furthermore, $Y \backslash Z$ has rational double points of fixed type along each connected component of $S$.

2. Assume that $P \in Y$ is an isolated Gorenstein singular point and that at least one irreducible component of $G:=b^{-1}(P)$ is normal. Then $G \simeq \mathbb{P}^{n}$ with normal bundle $\Omega_{\mathbb{P} n}^{1}$.

A key tool in the study of K3 surfaces is the Weyl group, the group generated by reflections with respect to the $(-2)$-classes in the Picard group (see [4] or [16]). Our conjectures imply that there is an analog in higher dimensions. For each $\rho \in E$ with square -2 , we obtain a reflection $s_{\rho}$ given by the formula

$$
s_{\rho}(v)=v+(v, \rho) \rho .
$$

The group $\mathcal{W}$ generated by these reflections is called the generalized Weyl group. Let $C_{+}(F, g)$ be the smallest real cone containing $\Lambda_{+}(F, g)$ and $\mathcal{D}(F, g)$ the subcone of $C_{+}(F, g)$ defined by $(v, \rho) \geq 0$ for each $\rho \in E$ with square -2 . This is a fundamental domain for the action of $\mathcal{W}$ on $C_{+}(F, g)$. The $(-10)$-classes in $E$ are walls for a subdivision of $\mathcal{D}(F, g)$ into subchambers. The interior of each subchamber is the ample cone for a symplectic birational 
model for $F$; the subchamber containing $g$ is the ample cone of $F$. In other words, $\mathcal{D}(F, g)$ is the closure of the birational ample cone of $F$.

One does not generally expect that the closure of the ample cone should be a fundamental domain for the action of a group $\mathcal{W}^{\prime} \supsetneq \mathcal{W}$. Algebraically, $(-10)$-classes do not yield reflections. Geometrically, one does not expect to find a group relating the various birational models of $F$. For a concrete example, see the discussion below of the Fano variety of lines on a cubic fourfold of discriminant eight.

We now describe the square-zero classes on the boundary of the closure of the ample cone:

CONJECTURE 3.8. - Let $\lambda$ be a primitive square-zero class on the boundary of the closure of the ample cone. Then the corresponding line bundle $\mathcal{L}=\mathcal{L}(\lambda)$ has no higher cohomology and its sections yield a morphism

$$
a: F \rightarrow \mathbb{P}^{2}
$$

whose generic fiber is an abelian surface.

The following theorem of Matsushita describes fiber space structures on holomorphic symplectic manifolds.

THEOREM 3.9. - ([19]) Let $F$ be a projective irreducible holomorphic symplectic manifold and $a: F \rightarrow B$ be a fiber space structure with normal base $B$ and generic fiber $F_{b}$. Then we have:

1. a is equidimensional in codimension-two points of $B$.

2. $K_{F_{b}}$ is trivial and there exists an abelian variety $\tilde{F}_{b}$ and an étale morphism $\tilde{F}_{b} \rightarrow F_{b}$.

3. $\operatorname{dim}(B)=n$ and $B$ has $\mathbb{Q}$-factorial log-terminal singularities.

4. $-K_{B}$ is ample and $B$ has Picard number 1.

5. The polarization on $B$ pulls back to a square-zero divisor on $F$.

Furthermore, if $F$ has dimension four then $F_{b}$ is an abelian surface.

Matsushita has also proved that such fibrations are Lagrangian [20]. For convenience, we provide a proof in our special case:

PROPOSITION 3.10. - In addition to the hypotheses of Theorem 3.9 assume that the dimension of $F$ is four. Then the fibers $F_{b}$ are Lagrangian. 
Proof. It suffices to prove this result for smooth fibers. Recall the formula for the Beauville form (see [13], 1.9)

$$
(\alpha, \alpha)=\int(\sigma \bar{\sigma}) \alpha^{2}-\left(\int \sigma \bar{\sigma}^{2} \alpha\right)\left(\int \sigma^{2} \bar{\sigma} \alpha\right),
$$

where $\sigma$ is the generator of $H^{0}\left(F, \Omega_{F}^{2}\right)$, normalized so that $\int(\sigma \bar{\sigma})^{2}=1$. If $\alpha$ is the pullback of the polarization on $B$ then the class of $F_{b}$ is equal to some rational multiple $c \alpha^{2}$. Since $\alpha$ is of type $(1,1)$, type considerations imply that the second term vanishes. On the other hand, the first term is equal to

$$
\left.\left.\frac{1}{c} \int_{F_{b}} \sigma\right|_{F_{b}} \bar{\sigma}\right|_{F_{b}} \text {. }
$$

Since $(\alpha, \alpha)$ is zero by the previous theorem it follows that $\sigma$ restricted to $F_{b}$ is also zero and therefore $F_{b}$ is Lagrangian (here we use the Hodge-Riemann bilinear relations).

REMARK 3.11. - It is instructive to compare Conjecture 3.8 with Wilson's results. Let $F$ be a Calabi-Yau threefold and $D$ a nef divisor class corresponding to a nonsingular point of the cubic hypersurface $\mu_{F}=0$ and satisfying $D c_{2}(F) \neq 0$. Then for some $n>0$ the linear series $|n D|$ is free and induces an elliptic fiber space structure on $F$ (see [28] $\S 3$ and [29] $\S 1$ ). The assumption $c_{2}(F) D \neq 0$ can be weakened under further technical hypotheses (see [30] and [31]).

REMARK 3.12. - Let $F$ be an irreducible holomorphic symplectic manifold and $\lambda \in \Lambda_{\text {nef }}(F)$ a divisor with $(\lambda, \lambda)=0$. Is $\lambda$ necessarily semiample?

\section{Deformation theory}

4.1. Deformations of subvarieties. - In this section, we work with arbitrary irreducible holomorphic symplectic manifolds.

THEOREM 4.2. - Let $F$ be an irreducible holomorphic symplectic manifold of dimension $2 n$ and $Y$ a submanifold of dimension $k$. Assume either that $Y$ is Lagrangian, or that all of the following hold: $\mathcal{N}_{Y / F}=\Omega_{Y}^{1} \oplus \mathcal{O}_{Y}^{\oplus 2 n-2 k}$, the restriction of the symplectic form to $Y$ is zero, and $H^{1}\left(\mathcal{O}_{Y}\right)=0$. Then the deformation space of $Y$ in $F$ is smooth of dimension $2 n-2 k$. 
The deformations of $F$ arising from deformations of the pair $(Y, F)$ are precisely those preserving the sub-Hodge structure

$$
\operatorname{ker}\left(H^{*, *}(F) \rightarrow H^{*, *}(Y)\right) .
$$

Proof. Identical to the proof of Corollary 3.4 in [24]. By [24], Corollary 3.2, any obstructions to deforming $Y$ in $F$ lie in the kernel of the natural projection

$$
\pi_{0,2}: H^{1}\left(N_{Y / F}\right) \rightarrow H^{1}\left(\Omega_{Y}^{1}\right) \otimes \operatorname{ker}\left(H^{0}\left(\Omega_{F}^{2}\right) \rightarrow H^{0}\left(\Omega_{Y}^{2}\right)\right)^{*} .
$$

Under our hypotheses this map is an isomorphism.

COROLlary 4.3. - Keep the hypotheses of theorem 4.2. Assume furthermore that the cohomology of $Y$ is generated by divisor classes. Then the deformations of $F$ arising from a deformation of the pair $(Y, F)$ are precisely those for which the image of

$$
H_{2}(Y, \mathbb{Z}) \rightarrow H_{2}(F, \mathbb{Z})
$$

remains algebraic.

4.4. Applications. - In this section we will assume that $F$ is deformation equivalent to $S^{[2]}$ for a K3 surface $S$. We show that the locus where our conjectures hold is open (in the analytic topology) in the moduli space. We will find points in the moduli space where our conjectures hold in subsequent sections.

THEOREM 4.5. - Let $F$ be as above and $R$ a nodal class on $F$. Assume that Conjecture 3.6 holds for $R$ and $F$. Let $F^{\prime}$ be a small projective deformation of $F$ such that $R$ deforms to a class $R^{\prime}$ of type $(3,3)$. Then the Conjecture remains true for $F^{\prime}$ and $R^{\prime}$.

Proof. Let $\pi: \mathcal{F} \rightarrow \Delta$ be a deformation of $F$ over a disc and $\mathcal{A}$ a nef and big line bundle on $\mathcal{F}$ such that $\mathcal{A}$ has degree zero on $R$. By Corollary 4.3, $R$ deforms to a family of rational curves $\mathcal{R} \subset \mathcal{F}$ over $\Delta$. In each fiber $\mathcal{R}_{t}$ itself deforms in a two parameter family. This family is parametrized by a deformation $\mathcal{S}_{t}$ of the $\mathrm{K} 3$ surface $S$ if $\rho$ is a $(-2)$-class. It is parametrized by a $\mathbb{P}^{2}$ if $\rho$ is a $(-10)$-class. Clearly, these are contracted by the sections of some power of $\mathcal{A}$. 
THEOREM 4.6. - Let $F$ be as above and $\lambda$ be a nef, square-zero divisor class on F. Assume that Conjecture 3.8 holds for $\lambda$ and F. Let $F^{\prime}$ be a small projective deformation of $F$ such that $\lambda$ deforms to a divisor class $\lambda^{\prime}$. Then the Conjecture remains true for $F^{\prime}$ and $\lambda^{\prime}$.

Proof. This is a consequence of Theorem 4.2 and the Lagrangian property proved in 3.10 .

4.7. Examples. - In this section we give examples of submanifolds satisfying the conditions of Theorem 4.2. Assume that $Y$ is a complete homogeneous space under a reductive algebraic group or a toric variety, and assume that the normal bundle to $Y$ is of the form stated above. Theorem 4.2 shows that the deformation space of $Y$ in $F$ is smooth. Moreover, the locus in the deformation space of $F$ corresponding to manifolds containing a deformation of $Y$ has codimension equal to the rank of the Néron-Severi group of $Y$. Here are some specific examples:

EXAMPLE 4.8. - Given $Y=\mathbb{P}^{n} \subset F$, the deformations of $F$ containing $\mathbb{P}^{n}$ form a divisor in the deformation space. For instance, if $S$ is a K3 surface containing a smooth rational curve $C$ then $C^{[n]} \simeq \mathbb{P}^{n} \subset S^{[n]}$. Let $R \in$ $H_{2}(F, \mathbb{Z})$ be the class of a line in $\mathbb{P}^{n}$ and $\rho \in H^{2}(F, \mathbb{Z})$ the corresponding divisor (i.e. $2 R v=(\rho, v)$ for $v \in H^{2}(F, \mathbb{Z})$ ). Then $\rho=2 C-e$ and $(\rho, \rho)=$ $-2(n+3)$.

EXAMPLE 4.9. - Again, let $S$ be a K3 surface containing a smooth rational curve $C$ and $F=S^{[n]}$. Consider the subschemes of $S$ of length $n$ with some support along $C$. The generic such subscheme is the union of a point of $C$ and a length $n-1$ subscheme disjoint from $C$. Thus we get a divisor $D_{1} \subset S^{[n]}$ birational to a $\mathbb{P}^{1}$ bundle over $S^{[n-1]}$. The normal bundle to a generic fiber $Y$ of this bundle is $\Omega_{Y}^{1} \oplus \mathcal{O}_{Y}^{\oplus 2 n-2}$. Let $R=[Y] \in H_{2}(F, \mathbb{Z})$ and $\rho=\left[D_{1}\right] \in$ $H^{2}(F, \mathbb{Z})$; note that $R$ corresponds to $\rho$ and $(\rho, \rho)=(C \cdot C)_{S}=-2$. The deformations of $S^{[n]}$ containing a deformation of $D_{1}$ are those for which $R$ (or $\rho$ ) remains algebraic. They have codimension one in the deformation space.

The relevance of these examples to Conjecture 3.6 is discussed in Section 5.7. We will give further examples in Section 6, where we consider cases where $F$ is deformation equivalent to $S^{[2]}$ and $Y=\mathbb{F}_{0}, \mathbb{F}_{1}$, or $\mathbb{F}_{4}$. We digress to give one further example that is particularly interesting: 
EXAMPLE 4.10. - We give a geometric realization of certain Mukai isogenies between K3 surfaces (cf. [21]). Let $S_{8}$ be a generic degree $8 \mathrm{~K} 3$ surface. In particular, we assume $S_{8}$ is realized as a complete intersection of 3 quadric hypersurfaces in $\mathbb{P}^{5}$, and the discriminant curve for these quadrics is a smooth sextic plane curve $B$. It follows that each such quadric $Q$ has rank five or six, and the corresponding family of maximal isotropic subspaces in $Q$ is parametrized by $\mathbb{P}^{3}$ or a disjoint union two copies of $\mathbb{P}^{3}$ respectively. As we vary $Q$, the families of maximal isotropic subspaces are parametrized by a K3 surface $S_{2}$ of degree 2, the double cover of $\mathbb{P}^{2}$ branched over $B$. Thus we obtain an étale $\mathbb{P}^{3}$-bundle $\mathcal{E} \rightarrow S_{2}$, mapping into $S_{8}^{[4]}$, with fibers satisfying the conditions of Proposition 4.2.

This yields an elegant universal construction of Brauer-Severi varieties representing certain 2-torsion elements of the Brauer group of a degree two K3 surface. Other 2-torsion elements are realized as étale $\mathbb{P}^{1}$-bundles $\mathcal{E} \rightarrow S_{2}$ arising from families of nodal rational curves (see the discussion of cubic fourfolds of discriminant 8 in Examples 7.8 and 7.15). The relationship between Mukai isogenies and Brauer groups is explored more systematically in the upcoming thesis of Caldararu [8].

\section{Symmetric squares of $\mathrm{K} 3$ surfaces}

Let $S_{2 n}$ be a K3 surface with Picard group generated by a polarization $f_{2 n}$ of degree $2 n$. The Beauville form restricted to the Picard group takes the form

\begin{tabular}{c|rr} 
& $f_{2 n}$ & $e$ \\
\hline$f_{2 n}$ & $2 n$ & 0 \\
$e$ & 0 & -2
\end{tabular}

The effective divisor with class $2 e$ is called the diagonal. It is isomorphic to a $\mathbb{P}^{1}$-bundle over $S_{2 n}$; the fibers are nodal rational curves. It follows that an ample line bundle has class of the form $x f_{2 n}-y e$ with $x, y>0$ and Proposition 2.1 implies that $2 n x^{2}-2 y^{2}>0$. The conjectures in Section 3 give sufficient conditions on $x$ and $y$ for $x f_{2 n}-y e$ to be ample.

Proposition 5.1. - Assume that $S_{2 n}$ is a $K 3$ surface with a polarization $f_{2 n}$ which embeds $S_{2 n}$ as a subvariety of $\mathbb{P}^{n+1}$. The line bundle a $f_{2 n}-e$ on $S_{2 n}^{[2]}$ is ample whenever $a>1$ or $a=1$ and $S_{2 n}$ does not contain a line. In particular, $f_{2 n}$ lies on the boundary of the closure of the ample cone. 
Proof. Let $S$ be a smooth surface embedded in projective space $\mathbb{P}^{r}$ and not containing a line. Then there is a morphism from the Hilbert scheme $S^{[2]}$ to the Grassmannian $\operatorname{Gr}(2, r)$. This morphism is finite onto its image. Therefore, the pullback of the polarization on the Grassmannian to $S^{[2]}$ is ample. We apply this to the image of $S_{2 n}$ under the line bundle $a f_{2 n}$.

REMARK 5.2. - In the event that $S_{2 n}$ does contain a line $\ell \subset \mathbb{P}^{n+1}$ the line bundle $f_{2 n}-e$ fails to be ample. However, it is nef and big and a sufficiently high multiple of it gives a birational morphism contracting the plane $\ell^{[2]}$ and inducing an isomorphism on the complement to this plane. In particular, there is a nodal $(-10)$-class $2[\ell]-e$ orthogonal to $f_{2 n}-e$.

5.3. Degree $2 \mathrm{~K} 3$ surfaces. - A K 3 surface $S_{2}$ of degree two can be realized as a double cover of $\mathbb{P}^{2}$ ramified in a curve of degree 6 . The quadratic form $2 x^{2}-2 y^{2}$ does represent -2 and -10 . The corresponding nodal classes are $e$ and $2 f_{2}-3 e$. The second class corresponds to the plane in $S_{2}^{[2]}$ arising from the double cover. Our conjectures predict that the ample cone consists of classes $x f_{2}-y e$ where $x, y>0$ and $2 x-3 y>0$. The quadratic form also represents 0 , but the corresponding class $f_{2}-e$ satisfies $\left(2 f_{2}-3 e, f_{2}-e\right)=-2$. After flopping the plane the proper transform of $f_{2}-e$ does yield an abelian surface fibration (the Jacobian fibration) - as expected.

Here is a sketch proof that the class $3 f_{2}-2 e$ is nef and big. Indeed, $3 f_{2}$ is very ample and embeds $S_{2}$ into $\mathbb{P}^{10}$. The image is cut out by quadrics $\mathcal{I}(2)$. Each pair of points on $S_{2}$ determines a line $\ell$. The quadrics vanishing on that line form a hyperplane in $\mathcal{I}(2)$. This induces a morphism from $S_{2}^{[2]}$ to $\mathbb{P}^{27}=\mathbb{P}\left(\mathcal{I}(2)^{*}\right)$ given by the sections of the line bundle.

5.4. Degree $4 \mathrm{~K} 3$ surfaces. - Let $S_{4}$ be a K3 surface with Picard group generated by a polarization of degree 4 . We take $f_{4}-e$ as the polarization of $S_{4}^{[2]}$. Now we describe the $(-2)$ and $(-10)$-lattice vectors in $\mathbb{Z} f_{4} \oplus \mathbb{Z} e$ and determine which are nodal classes. In fact, there are no $(-10)$-lattice vectors. The $(-2)$-vectors are of the form $\pm a_{m} f_{4} \mp b_{m} e$, where $a_{m} \sqrt{2}+b_{m}=$ $(2 \sqrt{2}+3)^{m}$. The vectors in the positive halfspace $\operatorname{Pic}_{+}\left(S_{4}^{[2]}, f_{4}-e\right)$ satisfy $2 x-y>0$. The nodal classes are $2 f_{4}-3 e$ and $e$. It is easy to see that all the other $(-2)$-classes in the positive halfspace are decomposable. We therefore predict that the ample cone is the interior of the cone spanned by 
$f_{4}$ and $3 f_{4}-4 e$. Indeed, $S_{4}^{[2]}$ has an involution exchanging $f_{4}$ and $3 f_{4}-4 e$ (given $p, q \in S_{4}$ the line spanned by $p$ and $q$ meets $S_{4}$ in two more points).

5.5. Degree $8 \mathrm{~K} 3$ surfaces. - Let $S_{8}$ be a K3 surface with Picard group generated by a polarization of degree 8 . This is the smallest degree case where there are no nodal classes besides the diagonal. Indeed, the quadratic form $8 x^{2}-2 y^{2}$ represents -2 and -10 exactly when $(x, y)=(0, \pm 1)$ and $( \pm 1, \pm 3)$. However, the parity condition for nodal classes of square -10 is not satisfied by $f_{8}-3 e$, i.e., $\left(f_{8}-3 e, H^{2}(F, \mathbb{Z})\right) \neq 2 \mathbb{Z}$. Therefore, our conjectures imply that the ample cone is the interior of the cone spanned by $f_{8}$ and $f_{8}-2 e$, and the second line bundle yields an abelian surface fibration $a: F \rightarrow \mathbb{P}^{2}$. We have already seen that the ample cone is contained in this cone. For an explicit construction of the abelian surface fibration, see [12] $\S 7$. There it is shown that the symmetric square of a generic K3 surface of degree $2 n^{2}(n>1)$ admits an abelian surface fibration.

REMARK 5.6. - This is a counterexample to the theorem in Section 2, p. 463 of [18]. There it is claimed that $S^{[2]}$ of a K3 surface $S$ admits a (Lagrangian) abelian surface fibration if and only if $S$ is elliptic.

5.7. K3 surfaces containing a rational curve. - Let $S$ be a K3 surface containing a rational curve $C$ and let $T$ be the surface obtained by blowing down $C$. Of course, $T$ has one rational double point. Consider the map $b: S^{[2]} \rightarrow \operatorname{Sym}^{2}(T)$. This map contracts rational curves corresponding to both $(-2)$ and $(-10)$-nodal classes.

The Hilbert scheme $S^{[2]}$ contains a plane $C^{[2]}$ and two distinguished divisors $D_{1}$ and $D_{2}$, birational to $\mathbb{P}^{1}$-bundles over $S$. The divisor $D_{1}$ is the locus of subschemes with some support in $C$ and $D_{2}$ is the diagonal. The map $b$ contracts $D_{1}$ and $D_{2}$ to surfaces isomorphic to $T$ and $C^{[2]}$ to the point $p$ where these surfaces intersect. The fiber $b^{-1}(p)$ is the union of $C^{[2]}$ and $\mathbb{F}_{4}$ (cf. Theorem 3.7).

The divisors $D_{1}$ and $D_{2}$ have classes $C$ and $2 e$ respectively. If $R$ is the class of a line in $C^{[2]} \simeq \mathbb{P}^{2}$ then the corresponding divisor class $\rho=2 C-e$. The $(-2)$-class $e$ and the $(-10)$-class $\rho$ are nodal; the class $C$ is not nodal. Of course, it becomes nodal upon flopping $C^{[2]} \subset S^{[2]}$. 


\section{Nonnodal smooth rational curves}

It is well known that for $\mathrm{K} 3$ surfaces all smooth rational curves are nodal and correspond to indecomposable (-2)-classes. In Section 5.7 we gave examples of nonnodal smooth rational curves; these curves were parametrized by a K3 surface. Here we discuss further examples of nonnodal smooth rational curves. As we shall see, these curves need not be parametrized by a K3 surface or a $\mathbb{P}^{2}$.

We first consider three examples where smooth rational curves do not correspond to nodal classes, but still correspond to classes with negative square. Let $F=S^{[2]}$, where $S$ is a K3 surface which is a double cover of a rational surface $\Sigma$ with Picard group of rank 2 . Then $F$ contains a surface isomorphic to $\Sigma$. We emphasize that the results of Subsection 4.1 apply in this case. This suggests certain refinements to Conjecture 3.6, which we formulate in each example.

EXAMPLE 6.1. - Let $S \rightarrow \Sigma=\mathbb{F}_{0}$ be branched over a general curve of type $(4,4)$. Hence the rulings induce two elliptic fibrations $E_{1}$ and $E_{2}$ which generate the Picard group and intersect as follows:

\begin{tabular}{r|rr} 
& $E_{1}$ & $E_{2}$ \\
\hline$E_{1}$ & 0 & 2 \\
$E_{2}$ & 2 & 0
\end{tabular}

Let $R_{1}$ and $R_{2}$ denote the rulings of $\Sigma \subset S^{[2]}$, with $\rho_{1}$ and $\rho_{2}$ their Poincaré duals in $\operatorname{Pic}\left(S^{[2]}\right)$. We have $\rho_{1}=E_{1}-e$ and $\rho_{2}=E_{2}-e$ so that the Beauville form may be written

\begin{tabular}{l|rr} 
& $\rho_{1}$ & $\rho_{2}$ \\
\hline$\rho_{1}$ & -2 & 0 \\
$\rho_{2}$ & 0 & -2
\end{tabular}.

Moreover, the $\rho_{i}$ generate a saturated sublattice of the Picard group and

$$
\left(\rho_{i}, H^{2}(F, \mathbb{Z})\right)=\mathbb{Z} .
$$

The smooth curves in the class $R_{1}+R_{2}$ move in a 3-parameter family on $\Sigma \subset F$. However, $\rho_{1}+\rho_{2}$ is not a nodal class. We conjecture that any holomorphic symplectic fourfold deformation equivalent to a symmetric square of a K3 surface with 2 nodal classes $\rho_{1}$ and $\rho_{2}$ as above should contain a surface $\Sigma=\mathbb{F}_{0}$. 
EXAMPLE 6.2. - Let $S \rightarrow \Sigma=\mathbb{F}_{1}$ be branched over a general curve of type $6 R_{0}+4 R_{-1}$, where $R_{0}$ is the class of the ruling and $R_{-1}$ is the class of the exceptional curve. The ruling induces an elliptic fibration $E$ on $S$ and the exceptional curve yields a rational curve $C \subset S$; these generate the Picard group and intersect as follows:

\begin{tabular}{r|rr} 
& $E$ & $C$ \\
\hline$E$ & 0 & 2 \\
$C$ & 2 & -2
\end{tabular}.

Let $\rho_{0}$ (resp. $\rho_{-1}$ ) be the Poincaré dual to $R_{0}$ (resp. $2 R_{-1}$ ). We have $\rho_{0}=E-e$ and $\rho_{-1}=2 C-e$, so that $\rho_{0}$ and $\rho_{-1}$ generate a saturated sublattice on which the Beauville form may be written

\begin{tabular}{c|rr} 
& $\rho_{0}$ & $\rho_{-1}$ \\
\hline$\rho_{0}$ & -2 & 2 \\
$\rho_{-1}$ & 2 & -10
\end{tabular}.

Moreover, $\left(\rho_{0}, H^{2}(F, \mathbb{Z})\right)=\mathbb{Z}$ and $\left(\rho_{-1}, H^{2}(F, \mathbb{Z})\right)=2 \mathbb{Z}$.

The smooth curves in the class $2 R_{0}+R_{-1}$ move in a 4-parameter family on $\Sigma \subset F$. However, $4 \rho_{0}+\rho_{-1}$ is not a nodal class. In this case we conjecture that any $F$ whose cohomology contains 2 nodal classes $\rho_{0}$ and $\rho_{-1}$ as above should contain a surface $\Sigma=\mathbb{F}_{1}$. Furthermore, we expect that $F$ is a specialization of a variety containing a plane $\Pi$ which corresponds to a $(-10)$-nodal class. This class is equal to $2 \rho_{0}+\rho_{-1}$ and $\Pi$ specializes to a union of a $\mathbb{P}^{2}$ and the $\mathbb{F}_{1}$ in $F$.

EXAMPLE 6.3. - Let $S \rightarrow \Sigma=\mathbb{F}_{4}$ be branched over the union of a general curve of type $12 R_{0}+3 R_{-4}$ and $R_{-4}$, where $R_{0}$ is the class of the ruling and $R_{-4}$ is the class of the exceptional curve. Again, the Picard group of $S$ is generated by an elliptic fibration $S$ and a rational curve $C$ which intersect as follows

$$
\begin{array}{r|rr} 
& E & C \\
\hline E & 0 & 1 \\
C & 1 & -2
\end{array} .
$$

Let $\rho_{0}$ (resp. $\rho_{-4}$ ) be the Poincaré dual to $R_{0}$ (resp. $R_{-4}$ ). Then we have $\rho_{0}=E-e$ and $\rho_{-4}=2 C+e$, so $\rho_{0}$ and $\rho_{-4}$ generate a saturated sublattice 
with Beauville form

\begin{tabular}{c|rr} 
& $\rho_{0}$ & $\rho_{-4}$ \\
\hline$\rho_{0}$ & -2 & 4 \\
$\rho_{-4}$ & 4 & -10
\end{tabular}.

Moreover, $\left(\rho_{0}, H^{2}(F, \mathbb{Z})\right)=\mathbb{Z}$ and $\left(\rho_{-4}, H^{2}(F, \mathbb{Z})\right)=2 \mathbb{Z}$.

The smooth curves in the class $5 R_{0}+R_{-4}$ move in a 7-parameter family on $\Sigma \subset F$. However, $5 \rho_{0}+\rho_{-4}$ is not a nodal class. In this case we conjecture that any $F$ with cohomology containing 2 nodal classes $\rho_{0}$ and $\rho_{-4}$ as above should contain a surface $\Sigma=\mathbb{F}_{4}$. We also expect that $F$ is a specialization of a variety containing a plane $\Pi$ which corresponds to a $(-10)$-nodal class. This class is equal to $4 \rho_{0}+\rho_{-4}$ and $\Pi$ specializes to a union of a $\mathbb{P}^{2}$ and the $\mathbb{F}_{4}$ in $F$.

Next we consider examples of smooth rational curves in $F$ where the corresponding class $\rho$ is of positive square.

EXAMPLE 6.4. - Let $S_{2}$ be a general K3 surface of degree 2 with polarization $f_{2}$. Let $C \subset S_{2}$ be a rational curve with two ordinary double points contained in the linear series $\left|f_{2}\right|$. Let $F \rightarrow \mathbb{P}^{2}$ be the compactified Jacobian for $\left|f_{2}\right|$. The fibers corresponding to $C$ are isomorphic to a product of nodal curves with normalization $\mathbb{P}^{1} \times \mathbb{P}^{1}$. Smooth curves of type $(1,1)$ in $\mathbb{P}^{1} \times \mathbb{P}^{1}$ yield smooth rational curves on $F$, deforming in a 3-parameter family. The homology class of these rational curves is double the class of the curve of type $(1,0)$, and is therefore not primitive.

EXAMPLE 6.5. - Let $S_{4} \subset \mathbb{P}^{3}$ be a general K3 surface of degree 4 and $f_{4}$ its polarization. Let $C \subset S_{4}$ be an elliptic curve in $\left|f_{4}\right|$ with two ordinary double points. Note that $C^{[2]}$ is a nonnormal ruled surface. Its fibers are smooth rational curves such that the corresponding class $\rho$ has square 2. Thus we get smooth rational curves in primitive homology classes such that the corresponding class $\rho$ has positive square as well.

As the rank of the Picard group of $F$ increases we expect more and more examples of nonnodal smooth rational curves parametrized by varieties of dimension $>2$.

REMARK 6.6. - Let $R \subset F$ be a smooth rational curve with primitive homology class. Then the Hilbert scheme of flat deformations of $R$ need not be 
irreducible and may have arbitrarily large dimension. Take $R \subset \mathbb{F}_{0} \subset F$ of bidegree $(1, n)$.

Question 6.7. - Assume that $\operatorname{rk} \operatorname{Pic}(F)=1$. Does there exist a smooth rational curve on $F$ ? Can we take its class to be primitive?

\section{Cubic fourfolds}

In this section, a cubic fourfold generally denotes a smooth cubic hypersurface $X \subset \mathbb{P}^{5}$. The variety $F$ parametrizing lines on $X$ is sometimes called the 'Fano variety of lines' - not to be confused with a variety with ample anticanonical class. It is known that $F$ is an irreducible holomorphic symplectic fourfold deformation equivalent to the Hilbert scheme of length-two subschemes of a K3 surface [1] [5]. Consequently, the conjectures of Section 3 apply. The existence of smooth rational curves $R \subset F$ translates into the existence of scrolls $T \subset X$. By definition, a scroll is the union of the lines parametrized by a smooth rational curve in the Grassmannian; it may have singularities. Our conjectures yield simple and verifiable predictions for the existence and nonexistence of scrolls in various homology classes of $X$. The presence of these scrolls yields unirational parametrizations of $X$ of various degrees.

7.1. Lattices, Nodal Curves, and Scrolls. - We recall standard facts about cubic fourfolds. We say that a cubic fourfold is special if it contains an algebraic surface not homologous to any multiple of the square of the hyperplane class $h^{2}$. Note that the intersection form $\langle$,$\rangle on the primitive cohomology$ takes the form

$$
\left(h^{2}\right)^{\perp} \simeq\left(\begin{array}{cc}
2 & 1 \\
1 & 2
\end{array}\right) \oplus_{\perp} U^{\oplus 2} \oplus_{\perp} E_{8}^{\oplus 2}
$$

(see [10], [9], [5]). Let $K=\mathbb{Z} h^{2}+\mathbb{Z T}$ be a saturated sublattice of algebraic classes in the middle cohomology of $X$. Then the discriminant $d=d(X, K)$ is the discriminant of $K$. It is a positive integer, congruent to 0 or 2 modulo 6 . The special cubic fourfolds of discriminant $d$ form an irreducible divisor $\mathcal{C}_{d}$ in the moduli space $\mathcal{C}$ of cubic fourfolds; $\mathcal{C}_{d}$ is nonempty iff $d>6$. For instance, $\mathcal{C}_{8}$ corresponds to the cubic fourfolds containing a plane $T_{1}$ and $\mathcal{C}_{14}$ corresponds to the cubic fourfolds containing a smooth quartic scroll $T_{4}$. 
The cohomology of a cubic fourfold and its Fano variety are closely related (see [5] for most of what follows). The incidence correspondence between $X$ and $F$ induces the Abel-Jacobi map

$$
\alpha: H^{4}(X, \mathbb{Z}) \rightarrow H^{2}(F, \mathbb{Z}),
$$

respecting the Hodge structures. We have that $\left(\alpha\left(h^{2}\right), \alpha\left(h^{2}\right)\right)=2\left\langle h^{2}, h^{2}\right\rangle$ and

$$
(\alpha(v), \alpha(w))=-\langle v, w\rangle
$$

for $v, w$ primitive. Note that $g:=\alpha\left(h^{2}\right)$ is the polarization on $F$ induced from the Grassmannian. The incidence correspondence induces a second map

$$
\beta: H^{6}(F, \mathbb{Z}) \rightarrow H^{4}(X, \mathbb{Z})
$$

respecting the Hodge structures. We can compose to obtain

$$
\psi: H_{2}(F, \mathbb{Z}) \rightarrow H^{6}(F, \mathbb{Z}) \stackrel{\beta}{\rightarrow} H^{4}(X, \mathbb{Z}) \stackrel{\alpha}{\rightarrow} H^{2}(F, \mathbb{Z}) \rightarrow H_{2}(F, \mathbb{Z}),
$$

where the first map is Poincare duality and the last map is induced by the Beauville form. We have $\psi(g)=2 g$ and $\psi(v)=-v$ for $v$ orthogonal to $g$.

Suppose that $F$ contains a smooth rational curve $R$ of degree $n$. Let $\tilde{T}$ be the universal line restricted to $R$ and $T \subset X$ the corresponding scroll sweeped out by $R$, which also has degree $n$. Note that the formula $\langle T, \Sigma\rangle=R \cdot \alpha(\Sigma)$ (for $\Sigma \in H^{4}(X, \mathbb{Z})$ ) follows from the incidence correspondence. Combining this with our computation of $\psi$, we obtain

$$
\langle T, T\rangle=R \cdot \alpha(T)=(R, \psi(R))=\frac{n^{2}}{2}-(R, R) .
$$

We use $T_{n, \Delta}$ to denote a scroll $T$ of degree $n$ for which the map

$$
\tilde{T} \rightarrow T \subset X
$$

has singularities equivalent to $\Delta$ ordinary double points (by definition, $\Delta$ is the number given by the double point formula). A Chern class computation gives

$$
\left\langle T_{n, \Delta}, T_{n, \Delta}\right\rangle=3 n-2+2 \Delta
$$

and we obtain the formula

$$
\Delta=\frac{1}{4}\left(n^{2}-6 n+4-2(R, R)\right) .
$$


The lattice generated by $h^{2}$ and $T_{n, \Delta}$ has discriminant

$$
\begin{aligned}
d(n, \Delta) & =3(3 n-2+2 \Delta)-n^{2}=6 \Delta-\left(n^{2}-9 n+6\right) \\
& =\frac{n^{2}}{2}-3(R, R) .
\end{aligned}
$$

This lattice has discriminant $>6$, so we obtain the lower bound

$$
\Delta \geq \Delta_{\min }(n):=\left\lceil\frac{1}{6}\left(n^{2}-9 n+6\right)+1\right\rceil .
$$

In particular, a cubic fourfold cannot contain smooth scrolls of degree $>7$.

REMARK 7.2. - The lattice $\mathbb{Z} h^{2}+\mathbb{Z} T_{n, \Delta}$ need not be saturated. For instance, if $n=8$ and $\Delta=5$ then $d(8,5)=32$. However, the lattice generated by $h^{2}$ and $T_{8,5}$ has index 2 in its saturation.

Proposition 7.3. - Let $X$ be a cubic fourfold, with Fano variety $F$. Let $R \subset F$ be a nodal rational curve and $T_{n, \Delta}$ the corresponding scroll. Then $\Delta$ takes the following values:

$$
\Delta=\left\{\begin{array}{l}
(m-2)(m-1) \text { if } n=2 m \\
(m-1)^{2} \text { and } m(m-2) \text { if } n=2 m+1 .
\end{array}\right.
$$

Proof. This is a consequence of Equations 1 and 2 above. We observe that $n$ is even when $(R, R)=-2$ and $n$ is odd when $(R, R)=-\frac{1}{2}$ or $-\frac{5}{2}$.

We summarize the numerical predictions for nodal scrolls of small degree in the following table:

\begin{tabular}{c|rrrrrrrrrrrrrr}
$n$ & 2 & 3 & 4 & 5 & 5 & 6 & 7 & 7 & 8 & 9 & 9 & 10 & 11 & 11 \\
\hline$\Delta$ & 0 & 0 & 0 & 0 & 1 & 2 & 3 & 4 & 6 & 8 & 9 & 12 & 15 & 16 \\
$d(n, \Delta)$ & 8 & 12 & 14 & 14 & 20 & 24 & 26 & 32 & 38 & 42 & 48 & 56 & 62 & 68
\end{tabular}

REMARK 7.4. - We can obtain cubic fourfolds containing scrolls $T_{n, \Delta}$ with more double points by exploiting nonnodal smooth rational curves on the corresponding Fano variety (see Examples 7.18 and 7.25). 
7.5. Unirational parametrizations. - We start with a classical example: if $X$ is a cubic fourfold containing a smooth quartic scroll $T_{4,0}$ then $X$ is rational. One would like to generalize this construction to other special cubic fourfolds.

Proposition 7.6. - Let $X$ be a cubic fourfold with Fano variety $F$. Assume that $F$ contains a smooth rational curve $R$ of degree $n$, with corresponding scroll $T_{n, \Delta}$. Assume that this corresponding scroll $T$ is not a cone. Then there exists a rational map

$$
\phi: \mathbb{P}^{4} \rightarrow X
$$

with

$$
\operatorname{deg}(\phi)=\left(\begin{array}{c}
n-2 \\
2
\end{array}\right)-\Delta=\frac{(n-2)^{2}}{4}+\frac{(R, R)}{2}+1 .
$$

Proof. Our assumptions imply that $R$ parametrizes pairwise disjoint lines in $X$. Given generic $\ell_{1}, \ell_{2}$, the cubic surface

$$
\operatorname{Span}\left(\ell_{1}, \ell_{2}\right) \cap X
$$

contains two disjoint lines and thus is rational. We therefore obtain a cubic surface bundle

$$
\begin{aligned}
& Y \stackrel{\sigma}{\rightarrow} \operatorname{Sym}^{2}(R) \simeq \mathbb{P}^{2} \\
& \begin{array}{l}
\phi \downarrow \\
X
\end{array}
\end{aligned}
$$

so that the fiber over the generic point contains two disjoint lines. Consequently, $Y$ is rational over $\mathbb{P}^{2}$ and thus is a rational variety.

To compute the degree of $\phi$, it suffices to compute the number of double points arising from a generic projection of the scroll $T$ into $\mathbb{P}^{4}$. The map $\tilde{T} \rightarrow \mathbb{P}^{4}$ has singularities equivalent to $\left(\begin{array}{c}n-2 \\ 2\end{array}\right)$ double points; $\Delta$ of these are from the singularities of $T$. We obtain the second formula for $\operatorname{deg}(\phi)$ by applying the Equation 2 of Section 7.1.

This demonstrates that the existence of rational curves in certain homology classes of $F$ implies that $X$ is rational. Unfortunately, our conjectures indicate that such rational curves are quite rare. If $R$ is nodal then $(R, R) \geq-\frac{5}{2}$, so $\operatorname{deg}(\phi)=1$ only when $n=4$ (see also Examples 7.12 and 7.21).

However, we obtain some interesting new unirational parametrizations of cubic fourfolds. Recall that in [10], the Fano variety of lines on the generic cubic fourfold of discriminant $2\left(N^{2}+N+1\right)(N>1)$ was shown to be 
isomorphic to $S^{[2]}$ of a $\mathrm{K} 3$ surface $S$. In particular, it contains nodal rational curves $R$ of degree $2 N+1$ with $(R, R)=-\frac{1}{2}$. One can show that the scroll corresponding to a generic such curve is not a cone, hence Proposition 7.6 applies. We obtain

$$
\operatorname{deg}(\phi)=N^{2}-N+1,
$$

which is always odd. In particular, the cubic fourfolds with odd degree unirational parametrizations are dense in the moduli space.

Cubic fourfolds are known to admit unirational parametrizations of degree two. Thus the cubic fourfolds described above admit unirational parametrizations of relatively prime degrees. There are few examples of irrational varieties with this property. Many common invariants used to detect irrationality (like the unramified cohomology of the function field) vanish in this situation.

7.7. Cubic fourfolds of small discriminant. - In this section we specialize our conjectures to Fano varieties of lines on general special cubic fourfolds of discriminant $d$. We obtain predictions on the existence and nonexistence of scrolls $T_{n, \Delta}$ on $X_{d} \in \mathcal{C}_{d}$. We verify these predictions in Section 7.14. Throughout we write $g=\alpha\left(h^{2}\right)$ and $\tau=\alpha(T)$.

EXAMPLE $7.8(d=8) .-$ For $X_{8} \in \mathcal{C}_{8}\left(\right.$ resp. $\left.F_{8}\right)$ we have intersection pairing (resp. Beauville form):

\begin{tabular}{c|rrr|rr} 
& $h^{2}$ & $T$ & & $g$ & $\tau$ \\
\hline$h^{2}$ & 3 & 1 & & $g$ & 6 \\
$T$ & 1 & 3 & & 2 & -2
\end{tabular},

so $\tau$ is a $(-2)$-class (note that $\left(\tau, H^{2}\left(F_{8}, \mathbb{Z}\right)\right)=\mathbb{Z}$.) There is also a $(-10)$ class: $\rho=g-2 \tau$. One can check that these classes are nodal. Therefore our conjectures predict a plane in $\Pi \subset F_{8}$ whose lines have degree one in the Grassmannian. This corresponds to a plane in $X_{8}$. They also predict a family of rational curves in $F_{8}$ parametrized by a $\mathrm{K} 3$ surface which correspond to quadric cones in $X_{8}$ (see Example 7.15).

This example illustrates our previous discussion concerning the action of the Weyl group. Here we have

$$
C_{+}\left(F_{8}, g\right)=\{a g-b \tau: 3 a+b>0, a-b>0\}
$$

and the fundamental domain for the action of the Weyl group is

$$
\mathcal{D}\left(F_{8}, g\right)=\{a g-b \tau: a+b \geq 0, a-b \geq 0\} .
$$


The conjectures predict that the ample cone should be

$$
\Lambda_{\mathrm{amp}}\left(F_{8}\right)=\{a g-b \tau: a+b>0, a-3 b>0\} ;
$$

the nef cone is bounded by clases of square 0 and 64 . If $F^{\prime}$ denotes the elementary transformation of $F_{8}$ along the plane $\Pi$, we expect

$$
\Lambda_{\mathrm{amp}}\left(F^{\prime}\right)=\{a g-b \tau: a-b>0,-a+3 b>0\} ;
$$

the nef cone is bounded by classes of square 8 and 64 . In particular, the two subchambers of $\mathcal{D}\left(F_{8}, g\right)$ are not conjugate.

EXAMPLE $7.9(d=12)$. - For $X_{12}$ and $F_{12}$ we have pairings:

$$
\begin{array}{c|ccc|cc} 
& h^{2} & T & & g & \tau \\
\hline h^{2} & 3 & 3 & & 6 & 6 \\
T & 3 & 7 & & 6 & 2
\end{array} .
$$

The $(-10)$-classes are given by $2 \tau-g$ and $3 g-2 \tau$. Our conjectures predict that $F_{12}$ contains two projective planes. The lines on these planes correspond to families of cubic scrolls on $X_{12}$ (see Example 7.16).

EXAMPLE $7.10(d=14)$. - For $X_{14}$ and $F_{14}$ we have pairings:

\begin{tabular}{c|rrr|rr} 
& $h^{2}$ & $T$ & & $g$ & $\tau$ \\
\hline$h^{2}$ & 3 & 4 & & 6 & 6 \\
$T$ & 4 & 10 & & 8 & 6
\end{tabular}

The nodal classes classes are given by $2 g-\tau$ and $2 \tau-g$. Note that $(2 g-$ $\left.\tau, H^{2}\left(F_{14}, \mathbb{Z}\right)\right)=\mathbb{Z}$ and $\left(2 \tau-g, H^{2}\left(F_{14}, \mathbb{Z}\right)\right)=2 \mathbb{Z}$. The first corresponds to a family of rational curves of degree 4 on $F_{14}$ parametrized by a K3 surface. The second corresponds to a family of rational curves of degree 5 also parametrized by a K3 surface (see Examples 7.17 and 7.19).

EXAMPLE $7.11(d=20)$. - For $X_{20}$ and $F_{20}$ we have pairings

$$
\begin{array}{l|rrr|rr} 
& h^{2} & V & & g & v \\
\hline h^{2} & 3 & 4 & g & 6 & 8 \\
V & 4 & 12 & v & 8 & 4
\end{array}
$$

where $v=\alpha(V)$. There are no $(-2)$-classes but there are two nodal $(-10)$ classes: $e_{1}=2 v-g, e_{2}=19 g-8 v$. The corresponding rational curves 
on $F_{20}$ have degrees 5 and 25, respectively (cf. Example 7.20). There is an involution interchanging $e_{1}$ and $e_{2}$ given by:

$$
\begin{array}{rlr}
g & \mapsto \begin{array}{r}
5 g-2 v \\
v
\end{array} \quad 12 g-5 v
\end{array} .
$$

\begin{tabular}{|c|c|c|c|c|}
\hline & $h^{2}$ & & & $\tau$ \\
\hline$h^{2}$ & & $g$ & f & 10 \\
\hline$T$ & $\begin{array}{ll}5 & 1\end{array}$ & $\tau$ & 10 & 8 \\
\hline
\end{tabular}

EXAMPLE $7.12(d=26)$. - For $X_{26}$ and $F_{26}$ we have pairings

This lattice does not represent -10 . The nodal $(-2)$-classes are $2 \tau-g$ and $109 g-38 \tau$. Note that

$$
\left(2 \tau-g, H^{2}\left(F_{26}, \mathbb{Z}\right)\right)=\left(109 g-38 \tau, H^{2}\left(F_{26}, \mathbb{Z}\right)\right)=2 \mathbb{Z} .
$$

Our conjecture predicts two families of rational curves parametrized by K3 surfaces, with degrees 7 and 137 respectively.

We next apply our conjecture on effective classes to derive the nonexistence of a $T_{5,2}$ on $X_{26}$. By Proposition 7.6, the existence of such a surface would imply the rationality of $X_{26}$. Let us assume that $T_{5,2} \subset X_{26}$ with ruling $R$. We may take $T$ for the class of $T_{5,2}$. We compute the class $\rho$ corresponding to $R$. Since

we get $\rho=5 g-2 \tau$. If we write

$$
\frac{1}{2} \rho \cdot g=5 \quad \frac{1}{2} \rho \cdot \tau=17
$$

$$
\rho=a(2 \tau-g)+b(109 g-38 \tau)
$$

then we find that $a=-7 / 45$ and $b=2 / 45$. This implies that $R$ is not contained in the (conjectured) monoid of effective classes. We shall show in Example 7.21 that a quintic scroll in $\mathbb{P}^{5}$ cannot have two double points.

QUESTION 7.13. - How can one systematize the argument for the nonexistence of $T_{5,2}$ 's on a (general) $X_{26}$ ? More precisely, let $X$ be a cubic fourfold containing a scroll $T_{n, \Delta}$ and assume that the lattice containing $h^{2}$ and $T_{n, \Delta}$ generates the lattice of algebraic classes in $H^{4}(X, \mathbb{Z})$. Do the values obtained in Proposition 7.3 give upper bounds for $\Delta$ in terms of $n$ ? 
7.14. Data. - In this section we present data from projective geometry concerning the existence of scrolls on cubic fourfolds. We organize the information by the degree of the scroll.

First of all, let us observe that a scroll of degree $n$ with ordinary double points can be obtained by projecting a smooth nondegenerate scroll of degree $n$ in $\mathbb{P}^{n+1}$ from a suitable linear subspace.

EXAMPLE $7.15\left(T_{2,0}\right)$. - Observe that a scroll of degree two cannot have ordinary double points at all. It is easy to see that the general cubic fourfold of discriminant 8 contains such a scroll. Furthermore, these scrolls are parametrized by a K3 surface of degree 2 (see [26], [10], [11]).

EXAMPLE $7.16\left(T_{3,0}\right)$. - A scroll of degree 3 also does not have any ordinary double points and it is contained in a general cubic fourfold of discriminant 12. On a fixed cubic fourfold these scrolls are parametrized by two disjoint $\mathbb{P}^{2}$ 's; given one scroll $T$, there is a residual scroll $T^{\prime}$ obtained by intersecting a linear and a quadratic hypersurface containing $T$ (see [9]). These correspond to two distinct $(-10)$-classes.

EXAMPLE $7.17\left(T_{4,0}\right)$. - A nondegenerate scroll of degree 4 in $\mathbb{P}^{5}$ does not have any ordinary double points. A general cubic fourfold of discriminant 14 contains a family of such scrolls, parametrized by a smooth K3 surface of degree 14. The corresponding class is a nodal (-2)-class (see, for example, [5], [9]).

EXAMPLE $7.18\left(T_{4,1}\right)$. - This example is closely related to Example 6.1. We will explain why the locus of cubic fourfolds containing a quartic scroll with one ordinary double point has codimension 2 in moduli. Consider a cubic fourfold $X$ containing such a scroll $T_{4,1}$. Note that $T_{4,1}$ is degenerate and is contained in a singular cubic threefold $Y$. We specialize first to the case where the quartic scroll degenerates to the union of two quadric scrolls. Each of these quadric scrolls is residual to a plane, and these planes intersect at a single point. What can we say about $Y$ in this case? A cubic threefold containing two such planes is obtained as follows. Let $C$ be a genus 4 stable curve obtained by taking a curve $C_{1}$ of type $(1,3)$ on a quadric surface $Q$, along with the union of two rulings $C_{2}$ and $C_{3}$ of type $(1,0)$. Note that $C$ is canonically imbedded in $\mathbb{P}^{3}$. Then $Y$ is the image of $\mathbb{P}^{3}$ under the linear 
series $|L|$ of cubics cutting out $C$. The planes in $Y$ are the total transforms of the lines $C_{2}$ and $C_{3}$. We claim that $Y$ contains a family of scrolls $T_{4,1}$, parametrized by $\mathbb{P}^{3}$. In particular, a cubic fourfold containing two planes meeting at a point also contains a three parameter family of $T_{4,1}$ 's. These are obtained by taking the proper transforms of the quadric surfaces $Z$ in $\mathbb{P}^{3}$ containing the lines $C_{2}$ and $C_{3}$. These form a linear series with projective dimension three. The restriction of $|L|$ to $Z$ is a linear series of type $(1,3)$ with two base points (i.e., the points of $Z \cap C_{1}$ not lying on $C_{2}$ or $C_{3}$ ). The image of $Z$ is a $T_{4,1}$.

This corresponds to a situation where $F$ contains a surface isomorphic to $\mathbb{P}^{1} \times \mathbb{P}^{1}$. The hyperplane sections give a 3 -parameter family of rational curves on $F$. As we have seen, such fourfolds should lie in codimension two (see 4.2).

EXAMPLE $7.19\left(T_{5,0}\right)$. - The cubic fourfolds of discriminant 14 also contain a family of quintic scrolls, parametrized by the same $\mathrm{K} 3$ surface which parametrizes the quartic scrolls. The corresponding class is a second nodal $(-2)$-class (see [5] or [9]).

EXAMPLE $7.20\left(T_{5,1}\right)$. - A general cubic fourfold $X_{20}$ of discriminant 20 contains a family of $T_{5,1}$ 's parametrized by a $\mathbb{P}^{2}$. It is known that $X_{20}$ contains a Veronese surface $V$. This also follows from Theorem 4.2 once we obtain a $\mathbb{P}^{2} \subset F_{20}$. The conic curves in $X_{20}$ lying in $V$ are parametrized by $\mathbb{P}^{2}$ as well. For each such curve $C$, let $H$ be the plane spanned by $C$ so that

$$
X_{20} \cap H=C \cup \ell
$$

where $\ell$ is a line. This yields a subvariety of $F_{20}$ isomorphic to $\mathbb{P}^{2}$; the lines $R \subset \mathbb{P}^{2}$ trace out $T_{5,1}$ 's on $X_{20}$. The corresponding $\rho \in \operatorname{Pic}\left(F_{20}\right)$ is a $(-10)$ class.

EXAMPLE $7.21\left(T_{5,2}\right)$. - There are no quintic scrolls with two ordinary double points in $\mathbb{P}^{5}$. (This is highly unfortunate because a cubic fourfold containing such a scroll would be rational by Proposition 7.6.) Let $\tilde{T}_{5,2} \subset$ $\mathbb{P}^{6}$ be the normalization and $p \in \mathbb{P}^{6}$ a point such that the projection of $\tilde{T}_{5,2}$ from $p$ is $T_{5,2}$. It follows that $\tilde{T}_{5,2}$ contains four coplanar points. However, 
these points necessarily lie on a conic curve $C \subset \tilde{T}_{5,2}$. This forces $T_{5,2}$ to be singular along the image of $C$.

EXAMPLE $7.22\left(T_{6,0}\right)$. - This remains to be explored - the corresponding $\rho$ is not nodal! The discriminant of the lattice $\mathbb{Z} h^{2}+\mathbb{Z} T_{6,0}$ is 12 .

EXAMPLE $7.23\left(T_{6,1}\right)$. - In this discriminant $(d=18)$ the Fano variety has two square-zero classes (bounding the ample cone, by our conjectures). In particular, there are no nodal classes in this case.

EXAMPLE $7.24\left(T_{6,2}\right)$. - The scroll $T_{6,2}$ is contained in a general cubic fourfold $X_{24}$ of discriminant 24. The family of such scrolls in a given cubic fourfold is parametrized by a K3 surface of degree 6 . The corresponding class $\rho$ is a nodal $(-2)$-class.

EXAMPLE $7.25\left(T_{6,3}\right)$. - The cubic fourfolds $X_{30}$ containing a sextic scroll with three ordinary double points are codimension 2 in moduli. The normalization $\tilde{T}_{6,3} \subset \mathbb{P}^{7}$ has 6 points lying in a 4-dimensional linear subspace, containing the line $\ell$ from which we project. These points necessarily are contained in a rational normal curve $C \subset \tilde{T}_{6,3}$ of degree 4 . The image of $C$ under projection is a quartic plane curve. This plane is necessarily contained in $X_{30}$ by Bezout's theorem, so $H^{2,2}\left(X_{30}, \mathbb{Z}\right)$ has rank at least 3 . Let us remark that the Fano variety $F_{30}$ contains a surface isomorphic to $\mathbb{P}^{1} \times \mathbb{P}^{1}$ and the rulings of the scrolls are given by type $(1,1)$-curves of this surface (cf. the discussion of $T_{4,1}$ and Example 6.1).

EXAMPLE 7.26 (Further examples). - Essentially the same argument shows that there are no scrolls $T_{6,4}$ (or $T_{6,5}$ or $T_{7,5}$ ): we look at the 8 points on the normalization $\tilde{T}_{6,4}$ spanning a 5-dimensional linear subspace containing the line of projection $\ell$. These points are necessarily contained on a rational normal curve of degree 5 on $\tilde{T}_{6,4}$. It projects to a quintic curve in $\mathbb{P}^{3}$ with 4 ordinary double points. This violates Bezout. 
BRENDAN HASSETT AND YURI TSCHINKEL

\section{References}

[1] A. Altman, S. Kleiman, Foundations of the theory of Fano schemes, Compositio Math. 34 (1977), no. 1, 3-47.

[2] A. Beauville, Some remarks on Kähler manifolds with $c_{1}=0$, Classification of algebraic and analytic manifolds (Katata, 1982), 1-26, Progr. Math., 39, Birkhäuser Boston, Boston, Mass., (1983).

[3] A. Beauville, Variétés Kähleriennes dont la premiére classe de Chern est nulle, J. Differential Geom. 18 (1983), no. 4, 755-782.

[4] A. Beauville, Préliminaires sur les périodes des surfaces K3, Géométrie des surfaces K3: modules et périodes (Exposé VII) (Palaiseau, 1981/1982), Astérisque No. 126 (1985), 91-97.

[5] A. Beauville, R. Donagi, La variété des droites d'une hypersurface cubique de dimension 4, C. R. Acad. Sci. Paris Sèr. I Math. 301 (1985), no. 14, 703-706.

[6] F. A. Bogomolov, The decomposition of Kähler manifolds with a trivial canonical class, (Russian) Mat. Sb. (N.S.) 93(135) (1974), 573-575.

[7] D. Burns, Y. Hu, T. Luo, Hyper-Kähler Manifolds and Birational Transformations in dimension 4, preprint (math.AG 0004154).

[8] A. Caldararu, Derived categories on varieties with trivial canonical bundle, Ph.D. Thesis, Cornell University (2000).

[9] B. Hassett, Special cubic hypersurfaces of dimension 4, Ph.D. Thesis, Harvard University, (1996).

[10] B. Hassett, Special cubic fourfolds, Compositio Math. 120 (2000), no. $1,1-23$.

[11] B. Hassett, Some rational cubic fourfolds, J. Algebraic Geom. 8 (1999), no. $1,103-114$.

[12] B. Hassett, Y. Tschinkel, Abelian fibrations and rational points on symmetric products, to appear in Internat. J. Math.

[13] D. Huybrechts, Compact hyper-Kähler manifolds: basic results, Invent. Math. 135 (1999), no. 1, 63-113.

[14] D. Huybrechts, The Kähler cone of a compact hyperkähler manifold, preprint (math.AG 9909109).

[15] Y. Kawamata, K. Matsuda, K. Matsuki, Introduction to the minimal model problem, Algebraic geometry, Sendai, 1985, 283-360, Adv. Stud. Pure Math., 10, North-Holland, Amsterdam-New York, 1987. 
[16] E. Looijenga, C. Peters, Torelli theorems for Kähler K3 surfaces, Compositio Math. 42 (1981), no. 2, 145-186.

[17] E. Markman, Brill-Noether duality for moduli spaces of sheaves on K3 surfaces, preprint (math.AG 9901072).

[18] D. G. Markushevich, Integrable symplectic structures on compact complex manifolds, Mat. Sb. (N.S.) 131(173) (1986), no. 4, 465-476; translation in Math. USSR-Sb. 59 (1988), no. 2, 459-469.

[19] D. Matsushita, On fibre space structures of a projective irreducible symplectic manifold, Topology 38 (1999), no. 1, 79-83.

[20] D. Matsushita, Addendum to: On fibre space structures of a projective irreducible symplectic manifold, preprint (math.AG 9903045) to appear in Topology.

[21] S. Mukai, Symplectic structure of the moduli space of sheaves on an abelian or K3 surface, Invent. Math. 77 (1984), 101-116.

[22] Y. Namikawa, Deformation theory of singular symplectic $n$-folds, preprint (math.AG 0010113) to appear in Math. Ann.

[23] V. Nikulin, Integral symmetric bilinear forms and some of their applications, Math. USSR Izvestija, 14 (1980), no. 1, 103-167.

[24] Z. Ran, Hodge theory and deformations of maps, Compositio Math. 97 (1995), no. 3, 309-328.

[25] N. Shepherd-Barron, Long extremal rays and symplectic singularities, preprint.

[26] C. Voisin, Théoréme de Torelli pour les cubiques de $\mathbb{P}^{5}$, Invent. Math. 86 (1986), no. 3, 577-601.

[27] J. Wierzba, Contractions of symplectic varieties, preprint (math.AG 9910130).

[28] P.M.H. Wilson, Calabi-Yau manifolds with large Picard number, Invent. Math. 98 (1989), no.1, 139-155.

[29] P.M.H. Wilson, The Kähler cone on Calabi-Yau threefolds, Invent. Math. 107 (1992), no. 3, 561-583. Erratum: Invent. Math. 114 (1993), no. 1, 231-233.

[30] P.M.H. Wilson, The existence of elliptic fibre space structures on CalabiYau threefolds, Math. Ann. 300 (1994), no. 4, 693-703.

[31] P.M.H. Wilson, The existence of elliptic fibre space structures on CalabiYau threefolds II, Math. Proc. Cambridge Philos. Soc. 123 (1998), no. 2, 259-262. 